\title{
Designing bowel preparation patient instructions to improve colon cancer detection
}

\author{
Evidence-based design criteria for patients' documents
}

Keywords: evidence, design, testing, iterating, human-centered

Medical personnel usually write and design documents that inform physicians or patients about procedures or therapies. Document design, however, requires skills that are not normally applied, resulting in information that is often not used properly. This article describes a project developed by the Alberta Colorectal Cancer Screening Program. The goal was to help patients better prepare for their colonoscopies. The process started with an analysis of the existing documents, and the development of performance specifications based on the literature on legibility, reading comprehension, memorization and use of information, plain language, visual perception, page layout, and image use. The project included an iterative process of prototyping and testing that resulted in 23 design criteria. Each iteration was tested with users to ensure ease of use, completeness of information, and accuracy and clarity to facilitate adoption. The project helped reduce practice variation regarding bowel preparation in the province of Alberta, Canada. This project illustrates how information design can help healthcare organizations provide patient-centred care. Information design helps patients engage in their own caring process, by providing information that people can use, understand and apply. After 15 months of use, the document has been downloaded more than 48,000 times, suggesting a good physician reception.

\section{Introduction: The medical and the design problem}

\subsection{The medical problem to be reduced}

Colorectal cancer is a leading cause of death. The accuracy of a colonoscopy depends, partly, on the quality of the bowel preparation. Poor bowel preparation results in cancelled colonoscopies, failed lesion detection, and repeated preparation and colonoscopy.

Inadequate preparation is common in patients, particularly in elderly patients and those with more than one chronic disease (Wexner et al. 2006).

Some of the reasons for poor bowel prep include: patient discomfort and inability to drink the required laxative volume, and the timing of the bowel prep (Ness, Manam, Hoen, \& Chalasani 2001). In addition, nurses receive different instructions from different physicians, leading to care variation. Research indicates that patients must understand what the colonoscopy involves, and why it is important (King-Marshall et al. 2016). Patient understanding of the importance of a clean colon 
and how to achieve this goal is key for a success-

ful colonoscopy.

To reduce practice variation, the Alberta Colorectal Cancer Screening Program commissioned Frascara-Noël to re-design three existing instructional documents to help patients prepare their intestines for colonoscopy. Each document was related to a different laxative currently in use.

\subsection{The problem of developing the bowel prep information tools}

\subsubsection{The users and the context in which the document is} used. In Alberta, Canada, the way in which the information is received can vary depending on the setting. If one is in a hospital of a large city, then most likely a nurse will go through the information with the patient; but if one is in a small clinic in a small town, one might need to go through the information without the help of a clinical expert. Hence, the document needs to be brief but complete, and as clear and accessible as possible.

Based on conversations with the Program's team, we generated the following question to guide our work: How can we convey information to help patients achieve an adequate bowel preparation?

To answer this question, we applied an EvidenceBased Practice design approach. According to Rousseau and Gunia (2016), "Evidence-based practice (EBP) is a disciplined approach to decision making and action, the hallmark of which is attention to evidence quality and the use of the best available evidence" (p. 668). Evidence-based practice is not restricted to research evidence published in scientific journals, but also includes specific information that the situation of implementation demands, stakeholders' requirements, and professional or practitioner experience (Rousseau \& Gunia 2016).

\section{Methods}

To develop the bowel prep instructions for patients we used a human-centered, evidence-based, and outcomesoriented approach to design (Frascara 2017).

Human-centered design is an approach that involves the users of the information tools at the beginning and throughout the design process. Human-centered design helps understand design problems from the perspectives of the users, and with the help of the users.

To involve users in the design process, we used thinking-aloud protocols and conducted ten face-toface interviews with patients, nurses, physicians and system administrators.

Thinking-aloud protocols (Frascara \& Noël 2010) help to better understand issues that users might encounter when reading: what is clear, what is not, what slows down reading, and what is looked at and when. We asked participants to read the text aloud and to tell us what came to their minds while reading. We gave participants a pencil to make notes on the document while performing the reading task.

For the face-to-face interviews, we developed a questionnaire to capture the clarity and accessibility of the tool, and people's thoughts and opinions about it (Lewis \& Ritchie 2003).

The interview had three main parts. The first part explored people's opinions about reading the new document prototype. The second part included a series of tasks to evaluate how easy it was to find information in the document, and to explore text comprehension and memory. For example, after reading the text participants were asked the following question: Can you please tell us how many days in advance of the colonoscopy you need to start preparing your bowel? In the third part participants were asked what they would change in the document. In this part, a 5-point Likert scale (1 being not easy to use and 5 being very easy to use) was 


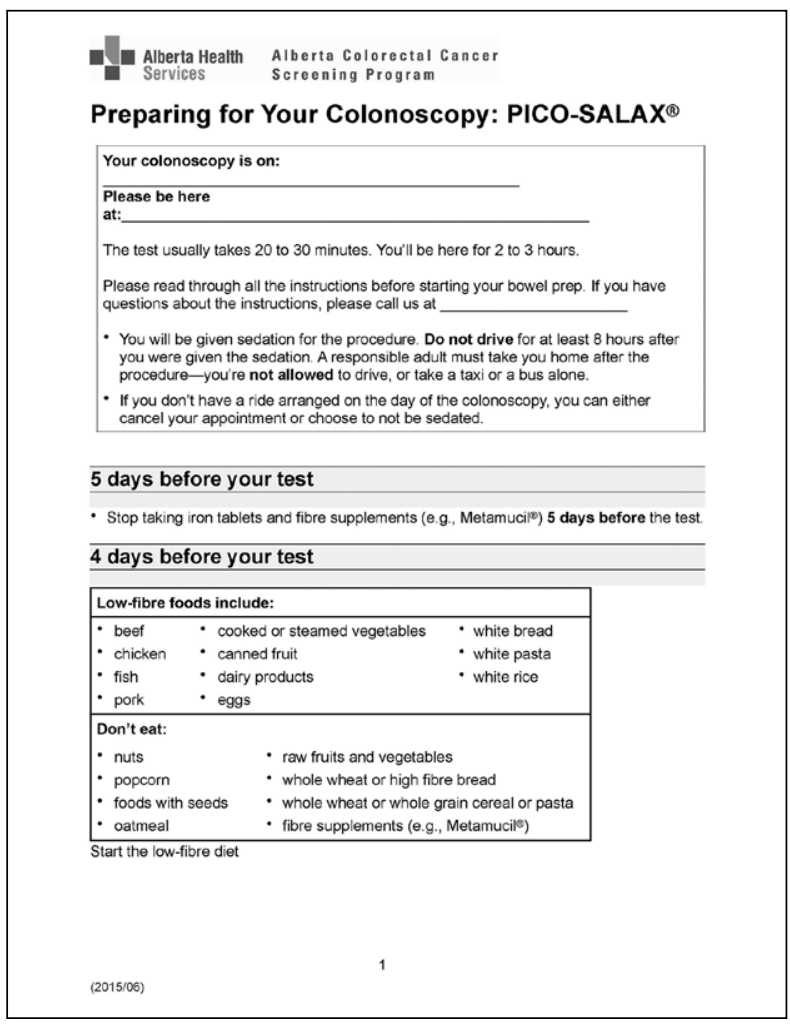

Figure 1. First page of the original 4-page document.

employed to evaluate the ease of use of the new document. Participants were asked the following question: Where would you place the document?

The interviews with the five clinicians took place in the participants' offices and lasted approximately one hour. The documents were sent to the participants in advance so that they could read them at their own pace.

We interviewed five patients: two who had had colonoscopies, and three who had never experienced a colonoscopy. All patients were over 65 years of age.

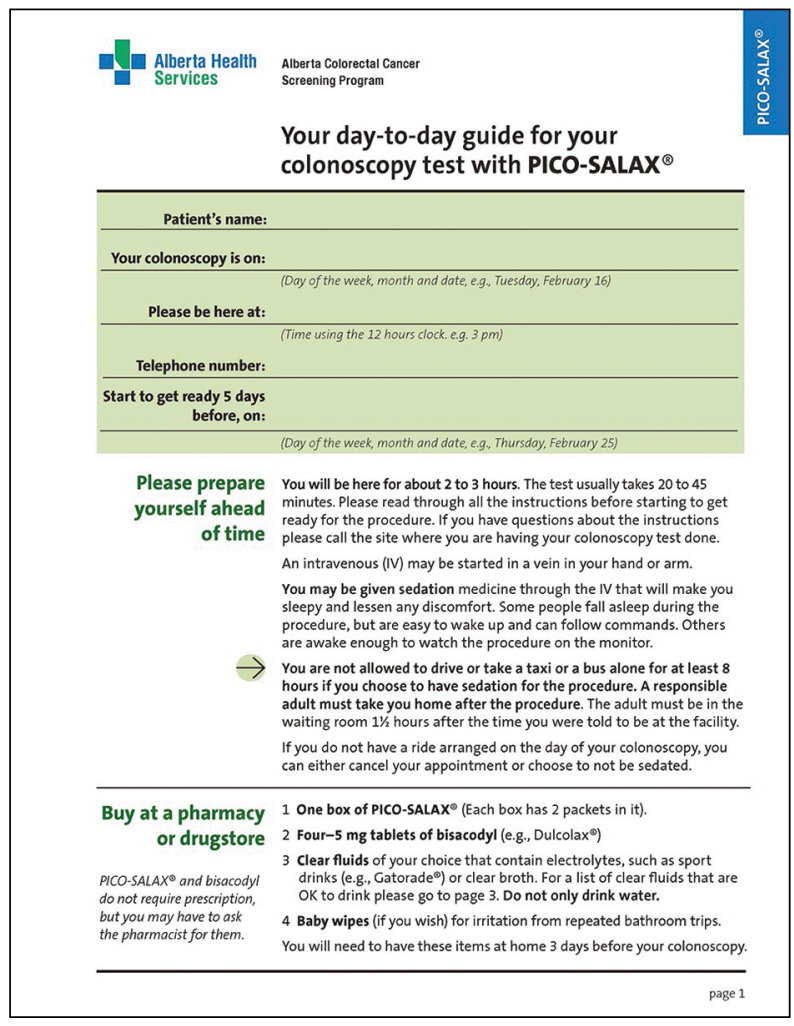

Figure 2. First page of the 7-page document redesign.

To gather scientific evidence with regard to the design of the documents we conducted a literature review. In general, our review focused on the following information design themes:

- Writing: prospect and framing, organizing text rhetorically, segmenting and grouping information, amount of content, sentence length and structure, tone, conditionals, and word length and frequency.

- Visual presentation of information: the notion of prospect, cognitive load, hierarchies; word recognition, 


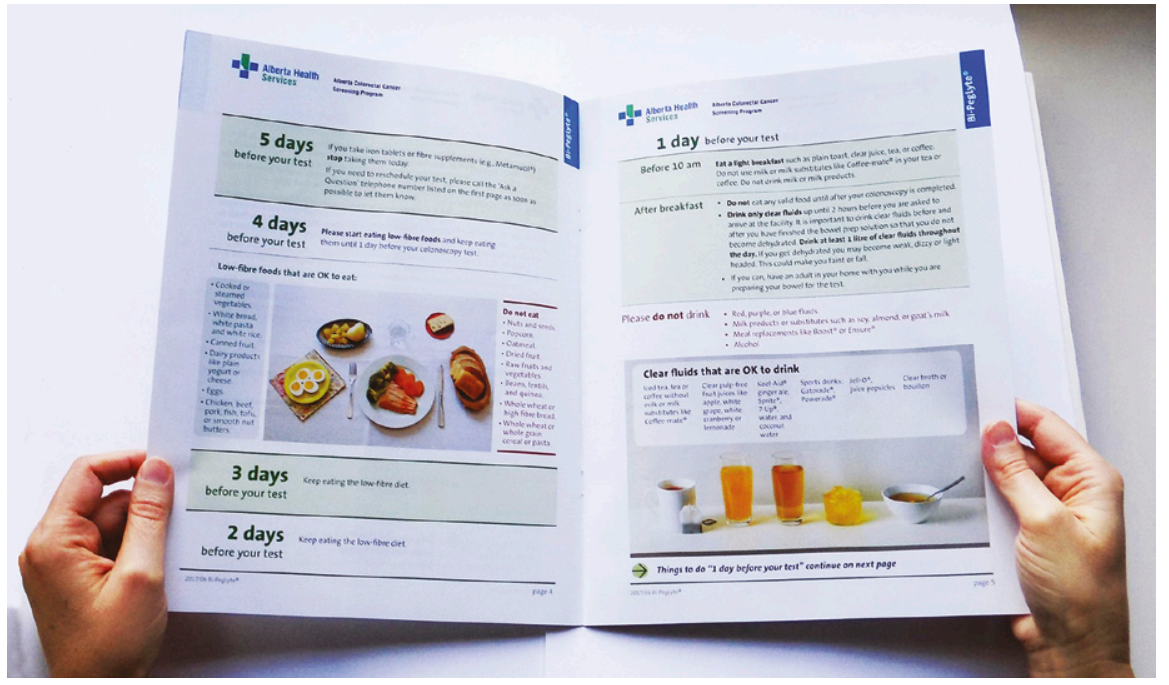

Figure 3. Double spread of the redesign - the text about food and drinks is now more visual. The new and better performing document proves that visual simplicity does not equal cognitive simplicity. type size and style to support reading, and other issues related to legibility such as column width, line spacing, and upper vs lower case letters;

- Text structure to support navigation and comprehension: the spatial arrangement of text, text organizers and signalling.

We surveyed peer-reviewed journals such as Applied Cognitive Psychology, Journal of Experimental Psychology, Ophthalmic Physiology Optics, Visible Language, and Information Design Journal. When an article was considered relevant, we appraised it to verify (a) whether the study addressed a clearly focused question, (b) how the study design helped address this question, (c) the level of rigour of the analysis, and (d) whether the data collected justified the conclusion and answered the research question. We also reviewed the work of expert authors in the information design community such as James Hartley, Richard Mayer, Karen Schriver, Patricia Wright, and Robert Waller.
This evidence was used to develop 23 design criteria to guide the design decisions with regard to the first prototypes. The content or clinical knowledge of the document was provided by the Alberta Colorectal Cancer Screening Program.

\subsection{Data analysis}

2.1.1 Thinking aloud protocols. In order to analyse the thinking aloud protocols and identify problematic areas, we aggregated all the participants' comments and the changes they proposed into a visual summary. We paired this visual information with the emotions noted while participants were reading aloud and when their reading was slowed down.

2.1.2 Face-to-face interviews. A deductive approach was used to analyse the answers provided in the first part of the interview, the questions provided a frame for aspects the authors needed to confirm or explore. 
For example: Is it easy to see and understand what the patient can eat?

In the second part, we measured how much time it took participants to find the answer, and the correctness of the answers. This helped to arrive at an average time necessary to find information.

In the third part of the interview, we collected data on reported ease of use for the existing document and for the redesign.

\section{Some of the design criteria developed based on scientific evidence}

The redesign focused on facilitating the reading task for patients who were preparing themselves for a colonoscopy. Facilitation of the reading task increases the likelihood that the intended public will read the texts and follow the recommendations. "Designing printed text with helpful formatting characteristics is advantageous in assisting people in acquiring information from printed materials" (Shaver \& Wogalter 2003: 830). Some of the design criteria and the references they are based on are presented below.

\subsection{Segmenting the information}

The information has to be segmented for understanding and learning purposes. Each segment's topic must be announced by a subtitle. "People learn better when a multimedia message is presented in learner-pace segments rather than as a continuous unit, people learn better from a multimedia message when they know the names and characteristics of the main concepts" (Mayer 2005: 6).

Evidence-based design criterion 1. The texts of a document must be segmented in cognitively affordable chunks, clearly labeled by subtitles and supported by layout.

\subsection{The notion of prospect}

Dixon (cited by Wright 1994) has shown that instructions are followed more accurately when they start by giving the reader a high-level description of the task. That is, subjects perform better regarding comprehension of instructions when a global description of the task is provided at the beginning rather than at the end of an explanation. "One way of promoting understanding is to clarify the goals that subsets of the instructions achieve" (Wright 1994: 18). Having a general idea of what one will confront improves one's ability to process information.

For this reason, titles and subtitles are very important, and they have to be reader-oriented, rather than topic-oriented. For instance, instead of writing "Patient information: Screening-related colonoscopy," we wrote "General points about your upcoming colonoscopy." Introducing the topic of a section is of great benefit to the reader. "Headings appear to provide a context which assists understanding" (Wright \& Barnard 1975: 216).

According to Lupker (2005), priming creates a semantic context that facilitates the recognition of the text to come. People tend to understand a word more quickly if a related concept is shown previously. Semantic priming supports aspects of perception and cognition, such as word recognition and text comprehension, by providing a context or framework that activates semantically related information.

Evidence-based design criterion 2. Titles that are readeroriented contribute to "priming" the reader in connection with the content of the ensuing text, and therefore facilitate understanding.

\subsection{Cognitive load: number of units of information}

George Miller (1994) discusses the "magical number seven" (plus two or minus two) as the key working 
memory capacity number. "I would propose to call this limit the span of absolute judgment, and I maintain that for unidimensional judgments this span is usually somewhere in the neighborhood of seven" (p. 90). He mentions several studies which propose that seven might be the largest number of items, e.g. locations, hierarchies or differences in a variety of scales that a person can remember with precision. While he proposes seven as a median, we are more inclined to take seven as a maximum, since we have to consider users with low literacy.

John Sweller (1988) was one of the first authors to develop a full theory of cognitive load. He discusses how "an increased number of statements in working memory increases cognitive load" (p. 265) and how overload creates difficulties for comprehending and learning.

Evidence-based design criterion 3. To ensure the cognitive affordability of the documents, they should have at most 7 parts, and each page should never have more than 4 sections, clearly separated by subtitles and other graphic devices.

\subsection{Type size and style to support legibility}

For comfortable reading, at 1 meter of reading distance, the height of lowercase letters should be between 3 and 4.5 millimeters, that is 2 to 3 times the size required for normal vision (Grether \& Baker 1972; Smith 1984).

Duncan and Konz (cited in Smith 1984) report that when subjects were asked to read numerals they approached them until they were 4.5 times the minimum required for error-free reading. Numerals require larger sizes because they do not form units such as words which can be recognized in a holistic manner (Smith 1984). It should be added that type size not only contributes to ease of reading, but also to memorization and use of textual information. As Waller (2011) explains, poor legibility of type slows readers down and affects reading accuracy.
Evidence-based design criterion 4. To support good legibility of texts, running text should have an $x$-height of $2 \mathrm{~mm}$ for $35 \mathrm{~cm}$ of reading distance. This is 3 times the minimum size for normal vision.

Evidence-based design criterion 5. Multi-digit numbers must match the height of capital letters to maintain the same ease of reading as the lower-case text.

\subsection{Type style}

Wogalter and Vigilante (2003) found that text with standard type widths was preferred over text with narrow type widths. The authors found that width affected reading speed, with narrower type resulting in significantly longer reading times.

Concerning stroke thickness, for a letter to maintain a good level of legibility, the thickness of its elements must be between one fifth and one eighth of the height of the character, and the character's width should be no less than three fifths of its height (Grether \& Baker 1972; Frascara 2006).

Evidence-based design criterion 6. Running texts should be set in medium weight, also called regular, plain or normal.

While the use of italics can be accepted as a way of calling attention in a subtle way to a few words within continuous prose, long texts in italics significantly reduce reading speed. Slattery and Rayner (2009) conducted an experiment using eye-tracking technology to compare the reading times when using regular texts, texts in italics and texts in bold. The authors reported that "readers took longer in general to read the target words in the italics" (p. 1145). A key aspect to consider when using italics is how steep the slope is. Hochuli (2008) recommends that "the slope should not be steeper than $10^{\circ}$ " (p. 21). 
Evidence-based design criterion 7. Italics should be used within texts where distinction is necessary; they are not to be used for emphasis or to help search-and-find tasks.

Tinker found that in some conditions "bold text type can be safely used for emphasis in... titles, section headings... without loss of legibility" (Tinker 1963: 62). A text might need emphasis on a word or two, to attract the reader to the main points of a paragraph. Bold text is a preferred option for this purpose, and the difference should be easily visible, particularly when bold text is used to facilitate the location of specific items in a long text.

Evidence-based design criterion 8. Bold type can be used within the text where there is a need for emphasis, to facilitate quick reference tasks, or for titles that need to be made more prominent to state their importance.

\subsection{Text structure and navigation to improve readability}

The connection between readability and comprehension is discussed extensively by George Klare (1984). Readability, rather than being associated with perception (as is legibility) is associated with cognition, and therefore reading performance varies dramatically between the different sectors of society, depending on educational level, lifestyle and daily reading activities.

The comprehension of texts (a cognitive act) is tinted by emotional tones (Frascara 1999; Sinclair 2002), and emotional tones are affected by aesthetic judgments.

\section{Evidence-based design criterion 9. Text composition} and layout should be designed to support both legibility and readability. The visual aspect of a document should be adapted to the aesthetic preferences of the users, since agreeable looks foster a good disposition in the user toward the document.

\subsection{The visual organization of a document}

Wogalter and Post (cited by Wogalter \& Vigilante 2003), "found that instructions in a list-type format produced better computer-task performance by experienced users than instructions that presented the same content in a prose-paragraph format" (p. 329). Other researchers recommend bulleted lists as superior to prose to draw attention to each key point while being brief (Hartley 2004). The American National Standard Institute (ANSI) concurs in recommending "outline format" and "outline with bullet format" as superior to "continuous format" to enhance readability (ANSI 2002). Desaulniers (1987) suggests that while subjects in an experiment spent similar amount of time reading information presented either in list or paragraph form, there might be a higher tendency to skim the text in a paragraph format. He states: "the paragraph layout increases the variability in reading behaviors, and the tendency to skim, rather than simply facilitate an increased reading rate" (p. 58). In another study it was found that the presentation of information as a flow chart led to easier learning and more compliance with the procedures. "The results indicate that performance was most accurate with the Flowchart format" (Desaulniers, Gillan \& Rudisill 1988: 291).

Evidence-based design criterion 10. The visual organization of a document - that is, giving the text a form that matches its content - enhances readability, leads to easier learning and to more compliance with the procedures.

Evidence-based design criterion 11. Lists tend to be more effective than continuous prose when the aim is to summarise and highlight information.

In a study discussing legal documents, it was "found that consent form comprehension was greater when... 
the form appeared to be more informal as compared to more official looking" (Wogalter, Howe, Sifuentes \& Luginbuhl 1999: 593). The same can be said of the tone of the text and the layout for the design of the bowel preparation instruction documents. All the above suggests that formatting can improve the ease of acquiring information, allowing readers to find the information more easily and more quickly.

Evidence-based design criterion 12. Writing instructional texts in a friendlier or more conversational way might be more effective than using formal or official language.

\subsection{Text organizers}

Panels, lines, and charts are devices that help readers understand the structure and argument of a document, and use the information strategically. Waller \& Waller (2015) use panels in the redesign of government letters. The authors stated "the use of panels also helps get over the problem that each panel contains text from a different source" (p. 214). Panels and lines are effective devices to visually group content in a rhetorically effective way, this means organizing the text "into meaningful groups that readers will notice, expect, and appreciate" (Schriver 2013: 390).

Evidence-based design criterion 13. Colour panels, lines and charts should be used to organise the text, to communicate the different parts of the document, identify sections and show how they are related.

\section{Findings}

The interviews and thinking aloud protocols generated new knowledge and understanding. For example, that evidence-based medicine is not a recipe, and needs to be adapted to each individual. We learned that physicians consider sentences starting with "Do not" condescending. They prefer the document to present first what needs to be done, and then what is to be avoided. Also, we identified that terms such as "average risk patients" need to be clearly defined. In addition, we found that marginalia and glossaries can be inconvenient when the reading task is one of quick reference.

Both designers and participants engaged in processes of thoughts not previously explored. This made it possible for participants to propose ideas to improve the documents which could not have been identified by the designers alone.

The analysis revealed problematic areas and the reasons why they were problematic. All interviewees clearly preferred the new versions of the documents over the existing ones. The images showing the meal and liquids were consistently praised. All participants agreed that the new document looked much better than its predecessor.

The quick reference tasks were efficient. Participants performed at a very high level in search-and-find tasks, finding specific targets very quickly (7.2 seconds average), despite the 7-page length of the document.

Regarding ease of use, participants rated the new document 4.75 on a 5 -point Likert scale, and the existing document 3.

Unexpectedly, during the interviews to keep gastroenterologists and family physicians focused on the visual presentation proved to be difficult. This was because they had strong opinions about certain aspects of the content. This led to an evaluation of their comments and reactions, and consequently to several changes. This resulted in a review of the content and of the design of the documents, extending the hours budgeted for this project by $40 \%$. For example, drinking restrictions were objected at this stage, and the new recommendation was that it should be 1 day before the colonoscopy, instead of 4 days. Similarly, to have a light breakfast (toast and tea) was now allowed for patients who have a colonoscopy 
appointment in the afternoon. "You don't want your patient to be weak," a gastroenterologist said.

The use of interviews after the thinking-aloud protocols was a very useful way of obtaining information about the participants' rationale for their thought processes.

Due to space limitations we describe here only 13 of the 23 evidence-based design criteria developed for this project to optimize decision-making in our design process. These criteria provided the basis for the design of the documents. This knowledge was combined with the learning from the thinking aloud protocols and interviews.

We developed 17 iterations, testing the documents with users and constantly refining them in consultation with content experts.

\section{Conclusion}

The performance of information documents does not hinge exclusively on their content and visual arrangement: it also depends on emotional reactions elicited and on the way the documents are used. Thus it is essential to generate trust.

During the interviews, potential patients showed a clear preference for the colour version of the new documents. The colour version appeared more trust-worthy. Preference substantially affects willingness to read, and willingness to read has a high correlation with adherence to procedures.

Patient-centred approaches to healthcare require a change from applying medical evidence-based information to cure a patient, to sharing information between researchers and healthcare providers, and between healthcare providers and patients, so that patients become active partners in the healing process. When patients are considered active partners in their healthcare, and are treated accordingly, they tend to better follow instructions and to experience better health outcomes.

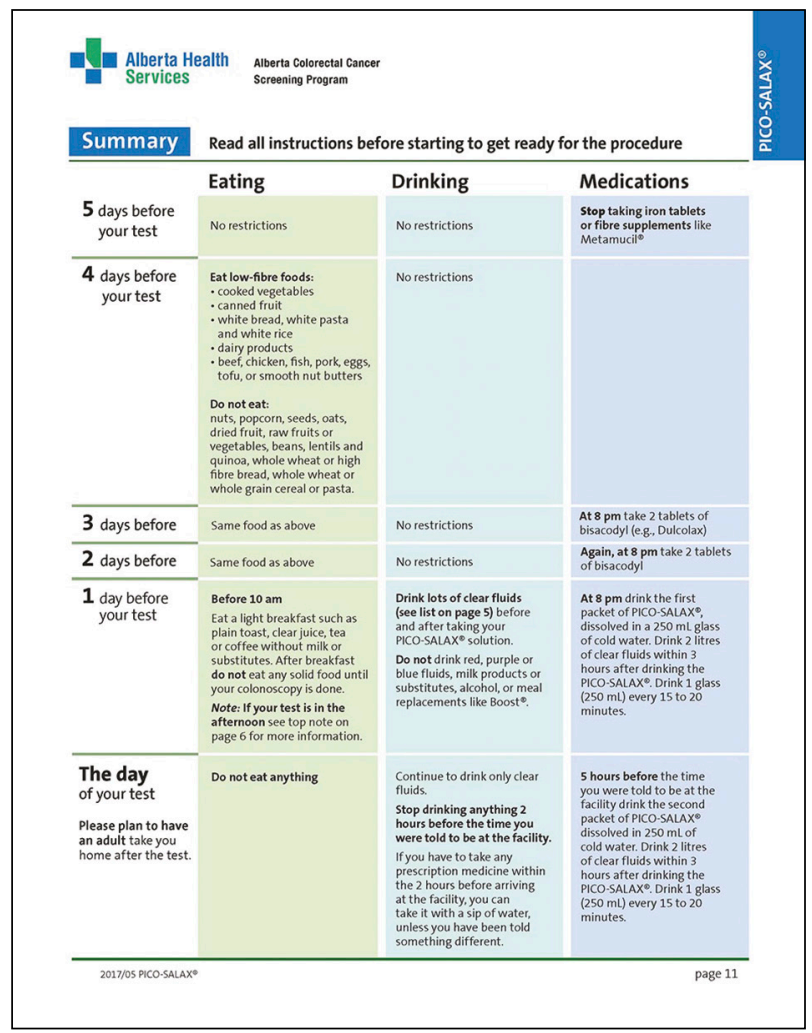

Figure 4. To aid memory and use, the redesign included a summary page. A patient said: "I would stick it on my fridge. Very helpful."

This is generated through a dialogical/conversational approach with the patients, rather than one where the health practitioners speak from a position of authority. It is with this in mind that we tried to develop the design of the documents.

Future revisions of existing documents could consider dividing the redesign process into two stages: first, engaging in a wide consultation with relevant knowledge experts, frontline care providers, and patients 
about the content of existing documents; and second, once the content had been agreed upon, moving on to the design of the visual presentation of the documents.

Renkema (cited in Janssen \& Neutelings 2001) listed four characteristics of government documents: impersonal, complex, diffuse, and traditional. Although in our project the design process took longer than expected, given the need to reconsider the documents' content, this resulted in more co-created documents: documents which are more personal, simpler, more concrete, and more pleasant. This helped to achieve patient-centred and clinician-centred health communications (Bodenheimer \& Sinsky 2014) for the Colorectal Cancer Screening Program at Alberta Health Centre. The new documents have been very well-received by the medical personnel. The documents became available in September 2017, and within one year the Alberta Colorectal Cancer Screening Program received more that 48,00o orders. An investigation to assess the impact of the new documents on patients who fail to prepare for the colonoscopy procedure appropriately is underway.

This article shows how an evidence-based approach to the design of documents can corroborate that the design profession is a reliable practice. Information design goes beyond being a craft. This approach facilitates the achievement of goals pursued in complex human interactions, where perceptual, cognitive, affective and behavioural factors play important roles.

\section{Submission date: 1 February, 2019 \\ Accepted date: 11 June, 2019}

\section{References}

American National Standard (2002). ANSI Z535.4-2002. American National Standard for product safety signs and labels. Retrieved from http://www.davis-inc.com/expert/docs/ z535p4-2002.pdf
Bodenheimer, T., \& Sinsky, C. (2014). From triple to quadruple aim: Care of the patient requires care of the provider. Analysis of Family Medicine, 12(6), 573-576. https://doi.org/10.1370/afm.1713

Desaulniers, D. R. (1987). Layout, organization and the effectiveness of consumer product warnings. Proceedings of the Human Factors and Ergonomics Society $31^{\text {st }}$ Annual Meeting (pp. 56-60). Santa Monica, CA: Human Factors and Ergonomics Society. https://doi.org/10.1177/154193128703100112

Desaulniers, D. R., Gillan, D. J., \& Rudisill, M. (1988). The effects of format in computer based procedure displays. Proceedings of the Human Factors and Ergonomics Society $32^{\text {nd }}$ Annual Meeting (pp. 291-295). Santa Monica, CA: Human Factors and Ergonomics Society. https://doi.org/10.1177/154193128803200511

Frascara, J. (1999). Cognition, emotion and other inescapable dimensions of human experience. Visible Language, 33(1), 74-89.

Frascara, J. (2006). Typography and the visual design of warnings. In M. Wogater (Ed.), The handbook of warnings (pp. 385-405). London, UK: Erlbaum.

Frascara, J., \& Noël, G. (2010). Evaluation and design of a blood components transfusion request form. Information Design Journal, 18(3), 241-249. https://doi.org/10.1075/idj.18.3.06fra

Frascara, J. (2017). Design, and design education: How can they get together? Art, Design \& Communication in Higher Education, 16(1), 125-131. https://doi.org/10.1386/adch.16.1.125_1

Grether, W. F., \& Baker, C. A. (1972). Visual presentation of information. In H. P. Van Cott \& R. G. Kinkade (Eds.), Human engineering guide to equipment design (pp. 49-127). Washington, DC: US Government Printing Office.

Hartley, J. (2004). Designing instructional and informational text. In D. H. Jonassen (Ed.), Handbook of research on educational communications and technology (pp. 917-947). Mahwah, $\mathrm{NJ}$ : Erlbaum.

Hochuli, J. (2008). Detail in typography. London: Hyphen Press. Janssen, D., \& Neutelings, R. (2001). Reading and writing public documents. Amsterdam, The Netherlands: John Benjamins.

Klare, G. R. (1984). Readability and comprehension. In R. Easterby \& H. Zwaga (Eds.), Information design (pp. 479-495). London, UK: Wiley \& Sons.

King-Marshall, E. C., Mueller, N., Dailey, A., Barnett, T. E., George Jr, T. J., Sultan, S., \& Curbow, B. (2016). "It is just another test they 
want to do": Patient and caregiver understanding of the colonoscopy procedure. Patient education and counseling, 99(4), 651-658. https://doi.org/10.1016/j.pec.2015.10.021

Lewis, J., \& Ritchie, J. (2003). Generalising from qualitative research. Qualitative research practice: A guide for social science students and researchers. London, UK: SAGE Publications.

Mayer, R. E. (2005). The Cambridge handbook of multimedia learning. Cambridge, NY: Cambridge University Press.

McNamara, T. (2005). Semantic priming: Perspectives from memory and word recognition. London, UK: Taylor \& Francis Group.

Miller, G. A. (1994). The magical number seven, plus or minus two: Some limits on our capacity for processing information. Psychological Review, 101(2), 343-352.

Ness, R. M., Manam, R., Hoen, H., \& Chalasani, N. (2001). Predictors of inadequate bowel preparation for colonoscopy. The American journal of gastroenterology, 96(6), 1797. https://doi.org/10.1111/j.1572-0241.2001.03874.x

Rousseau, D. M., \& Gunia, B. C. (2016). Evidence-based practice: the psychology of EBP implementation. Annual Review of Psychology, 67, 667-692. https://doi.org/10.1146/annurev-psych-122414-033336

Slattery, T. J., \& Rayner, K. (2009). The influence of text legibility on eye movements during reading. Applied Cognitive Psychology, 24(8), 1129-1148. https://doi.org/10.1002/acp.1623

Smith, S. (1984). Lettersize and legibility. In R. Easterby \& H. Zwaga (Eds.), Information design (117-186). Chichester, UK: Wiley.

Schriver, K. (2013). What do technical communicators need to know about information design? In J. Johnson \& S. Selber (Eds.), Solving problems in technical communication (pp. 386-427). Chicago, IL: University of Chicago Press.

Shaver, E. F., \& Wogalter, M. S. (2003). A comparison of older vs. newer over-the-counter (OTC) nonprescription drug labels on search time accuracy. Proceedings of the Human Factors and Ergonomics Society $47^{\text {th }}$ Annual Meeting (pp. 826-830). Santa Monica, CA: Human Factors and Ergonomics Society.

Smith, S. L. (1984). Letter size and legibility. In R. Easterby \& H. Zwaga (Eds.), Information design (171-186). London: Wiley.

Sweller, J. (1988). Cognitive load during problem solving: Effects on learning. Cognitive Science, 12(2): 257-285. https://doi.org/10.1016/0364-0213(88)90023-7
Tinker, M. A. (1963). Legibility of print. Ames, IA: lowa University Press.

Waller, R. (2011). What makes a good document? The criteria we use. Reading, UK: University of Reading. Retrieved from http://www.reading.ac.uk/web/files/simplification/ sc2criteriagooddoc-7.pdf

Waller, R., \& Waller, J. (2015). Transforming government letters: Design and writing working together. In J. Frascara (Ed.), Information design as principled action: Making information accessible, relevant, understandable, and usable (210-222). Champaign, IL: Common Ground.

Wexner, S. D., Beck, D. E., Baron, T. H., Fanelli, R. D., Hyman, N., Shen, B., \& Wasco, K. E. (2006). A consensus document on bowel preparation before colonoscopy: Prepared by a task force from the American Society of Colon and Rectal Surgeons (ASCRS), the American Society for Gastrointestinal Endoscopy (ASGE), and the Society of American Gastrointestinal and Endoscopic Surgeons (SAGES). Surgical Endoscopy and Other Interventional Techniques, 20(7), 1147-116o. https://doi.org/10.1016/j.gie.2006.03.918

Wogalter, M. S., Howe, J. E., Sifuentes, A. H., \& Luginuhl, J. (1999). On the adequacy of legal documents: factors that influence informed consent. Ergonomics, 42(4), 593-613. https://doi.org/10.1080/001401399185504

Wogalter, M. S., \& Vigilante, W. J. Jr., (2003). Effects of label format on knowledge acquisition and perceived readability by younger and older adults. Ergonomics, 46(4), 327-344. https://doi.org/10.1080/0014013021000048006

Wright, P. (1994). Enhancing the usability of written instructions. Proceedings of Public Graphics (pp. 26-30). Lunteren, The Netherlands: Delft University and Utrecht University.

Wright, P., \& Barnard, P. (1975). 'Just fill in this form': A review for designers. Applied Ergonomics, 6(4), 213-220. https://doi.org/10.1016/0003-6870(75)90113-1

\section{About the authors}

Dr. Guillermina Noël is a design researcher and educator. Guillermina is the new Head of the bachelor "Design Management, International" at the Lucerne University of Applied Sciences and Arts (Hochschule

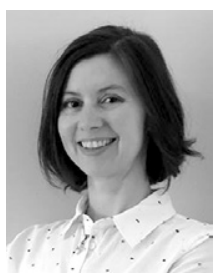


Luzern), Switzerland. She applies a human-centred, evidencebased, and outcomes-oriented design approach to improve healthcare. For more than two years Guillermina was a Research Academic, at the Faculty of Medicine \& Dentistry, University of Alberta, Canada. In this role, Guillermina worked with healthcare teams trying to improve care practices (Quality Improvement) or transferring and implementing health research into health practice to influence every day health decisions (Knowledge Translation). As a human-centred designer, she supported healthcare teams by helping them to understand the clinicians' and patients' needs, integrating their requirements with those of the stakeholders, and co-designing tools seeking to achieve impact. She is the Director of the Health Design Network. She is member of the Editorial Board of Information Design Journal.

Email: fnoel@ualberta.ca

Jorge Frascara is Professor Emeritus and former Chairman, Art \& Design, University of Alberta; Honorary Professor, Emily Carr University; Fellow Society of Graphic Designers of Canada; Former-President of Ico-D (International Council of Design); Advisor, Design Doctorate, IUAV University of Venice, Advisory Board Member Master Design Hochschule Luzern, and Editorial Board Member of Visible Language, Design Issues, She-Ji, and Information Design Journal. Jorge is the author of 11 books, including Information Design as Principled Action (Common Ground 2015), and Enseñando Diseño (Ediciones Infinito 2018). He has published more than 90 articles. He guest edited a special issue of Visible Language $(49 / 1-2,2015)$ on Design and Health. He has been advisor to the International Standards Organization (ISO), the
Canadian Standards Association and the Canadian Standards Council on public information symbols. He gave lectures in 26 countries, and received honours for his socially-oriented practice and promotion of communication design from eight countries. Past clients include the Government of Canada, the Government of Alberta, the Mission Possible Coalition: Traffic safety, the Alberta Drug Utilization Program, Alberta Health Services, and the Emilia Romagna Health Services, Italy. He now lives in Lucerne, Switzerland, consulting on information design related to health and education.

Email: frascara@ualberta.ca

Dr. Clarence Wong is an Associate Professor with the Division of Gastroenterology at the University of Alberta. His key academic and clinical focus is therapeutic endoscopy for gastrointestinal cancers. He is the medical director of the Edmonton Endoscopic Ablation Program which treats Barrett's esophagus and early upper gastrointestinal

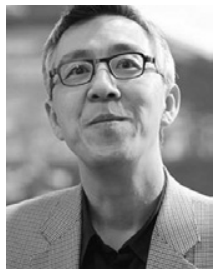
tract cancers. He is also the provincial medical director of the Alberta Colorectal Cancer Screening program (ACRCSP). His research interests include clinical and laboratory innovations in colon cancer screening, quality in endoscopy and Barrett's esophagus. He has received regional and national teaching awards for excellence in medical education including the University of Alberta Medical Students' Association Teacher of the Year Award, the Canadian Association of Medical Education (CAME) Certificate of Merit, and the University of Alberta Rutherford Award for Excellence in Undergraduate Teaching.

Email: ckw3@ualberta.ca 